\title{
Die Wirkung des Adrenalins auf das in der Leber gespeicherte Eiweiß.
}

\author{
Von \\ Hans Stïbel.
}

(Aus der physiologisehen Anstalt der Universität Jena.)

Mit 3 Textabbildungen.

(Eingegangen am 9. August 1920.)

Bereits vor einer Reihe von. Jahren konnte $\mathrm{Berg}^{1}$ ) auf mikrochemischem Wege den Nachweis erbringen, daß in der Leber bei reichlicher Ernährung mit Eiweißkörpern eine Speicherung von Eiweiß eintritt. Berg fand einen charakteristischen Unterschied im Verhalten der Leberzellen vor allem beim Salamander und Kaninchen, wenn er einerseits stark mit Eiweiß gefütterte Tiere, andererseits nicht mit Eiweiß gefütterte oder Hungertiere untersuchte. Der Unterschied besteht darin, daß beim mit Eiweiß gefütterten Tier die Leberzellen mit ziemlich großen, tropfenförmigen Gebilden erfüllt sind, die sich besonders schön durch Färbung von Schnittpräparaten mit Methylgrün-Pyronin darstellen lassen, da sie durch Pyronin leuchtend rot gefärbt werden, während der übrige Zelleib farblos bleibt oder nur eine schwache Rotfärbung annimmt. Diese Gebilde zeigen auch die Millonsche Reaktion. An Präparaten von Hungertieren oder von solchen Tieren, die nicht reichlich mit Eiweiß gefüttert wurden, sind diese durch Pyroninfärbung darstellbaren Körper nicht wahrnehmbar. Berg konnte auch nachweisen, daß das Auftreten und Verschwinden der mit Pyronin färbbaren Körper nicht der Ausdruck verschiedener durch Gallenabsonderung bedingter Tätigkeitszustände der Leber ist. Berg und Cahn - Bronner ${ }^{2}$ ) haben dann weiterhin gezeigt, daß man durch Fütterung mit vollständig abgebautem Eiweiß bzw. mit Aminosäuregemischen die Entstehung dieser Körper gleichfalls hervorrufen kann.

Ich habe in einer Reihe von Versuchen die Angaben Bergs nachgeprüft und kann dieselben, insbesondere soweit es sich um Versuche an der Ratte handelt, durchaus bestätigen. Die ersten Versuche, die ich. am Frosch und am Kaninchen anstellte, lieferten negative Ergebnisse. Die Untersuchungen am Frosch wurden in der Weise angestellt, daß die

1) W. Berg, Anatomischer Anzeiger 42, 251. 1912; Biochem. Zeitschr. 61, 428. 1914.

2) W. Berg und C. Cahn-Bronner, Biochem. Zeitschr. 61, 434. 1914. 
Versuchstiere - mittelgroße Exemplare von Rana temporaria -8 bis 14 Tage lang täglich mit $1 / 2-1$ g Froschfleisch gefüttert wurden, indem ihnen das Fleisch in den Oesophagus eingeschoben wurde. Es wurde sodann ein Stück der Leber zur mikroskopischen Untersuchung verwendet und mit der Leber eines nicht gefütterten Kontrolltieres verglichen. Ein Unterschied zwischen gefüttertem Tier und Kontrolltier ließ sich nicht feststellen. Diesem negativen Ergebnis ist schon deshalb wenig Bedeutung beizumessen, als die Versuche im Winter vorgenommen wurden, zu einer Zeit, in der normalerweise der Frosch überhaupt keine Nahrung aufnimmt. - Ebenso negativ verliefen analoge Versuche an zwei Kaninchen, die eine Woche lang täglich $10 \mathrm{~g}$ Nutrose (in wässeriger Lösung, mittels Schlundsonde) und daneben noch reichlich Rüben erhalten hatten. Auch hier zeigte sich am mikroskopischen Präparat der Leber kein Unterschied zwischen mit Eiweiß gefüttertem und normal gefüttertem Kontrolltier, indem die von Berg beschriebenen mit Pyronin färbbaren Körper in keinem Falle gefunden werden konnten. Von weiteren Untersuchungen an Kaninchen, die sich nach Berg besonders gut zum Nachweise der Eiweißspeicherung in der Leber eignen, mußte aus Mangel an Tiermaterial abgesehen werden.

Gegenüber diesen negativen Ergebnissen erwies sich nun die weiße zahme Ratte als besonders geeignet zur Wiederholung und Bestätigung der von Berg angestellten Versuche. Es wurden Präparate von Tieren, die vorwiegend mit Semmel und von Tieren, die vorwiegend mit Fleisch gefüttert worden waren, und weiterhin von Hungertieren miteinander verglichen. Die Fleischfütterung wurde stets mindestens eine Woche lang durchgeführt, und zwar wurde den betreffenden Versuchstieren neben möglichst reichlichen Fleischmengen stets noch etwas Semmel und häufig auch Speck verabreicht.

Von der Leber wurden kleine Stücke entsprechend den Angaben von Berg in Zenker -Formol (Zenkersche Flüssigkeit $+10 \%$ Formaldehyd. sol.) und andere Stücke in Alkohol absolutus fixiert. Die mikroskopischen Bilder, die mit Hilfe dieser beiden Fixierungsmethoden erhalten wurden, unterschieden sich nicht voneinander. Es wurde deshalb in späteren Versuchen nur noch die Fixierung mit Alkohol absolutus (12 bis 24 Stunden lang) angewendet, da nach Alkoholfixierung die Einbettung in Paraffin erheblich rascher vollzogen werden kann. Von den Paraffinblöcken wurden $5 \mu$ dicke Schnitte hergestellt. Zur Färbung wurde ein Gemisch von Methylgrün und Pyronin in wässeriger Lösung verwendet ${ }^{1}$ ). Die Schnitte blieben etwa 1 Stunde lang in dem Farbge-

$\left.{ }^{1}\right)$ Lösung I: Methylgrün 1,0, Acid. carbolic. liquefact. 0,25, Aq. dest. 100,0; Lösung II: Pyronin. pur. 1,0, Acid. carbolic. liquefact. 0,25, Aq. dest. 100,0. 15 Teile von I werden mit 35 Teilen von II gemischt (nach Grawitz aus Weidenreich, „Blut" in Enzyklopädie der mikroskopischen Technik, 2. Aufl., 1910, Bd. I, S. 129. 
misch. Hieranf erfolgte eine kurze Differenzierung in 70 proz. Alkohol, die unter dem Mikroskop nachgeprüft wurde. Da die Differenzierung sehr rasch eintritt, muß vor der mikroskopischen Prüfung der 70 proz. Alkohol erst wieder durch destilliertes Wasser abgespült werden. Zur Beobachtung der Differenzierung leistete mir die neue Zeisssche Wasserimmersion Q, num. Apert. 0,85, 90 fache Objektivvergrößerung, besonders gute Dienste. Nach ganz kurzem Abspülen des sorgfältig mit Fließpapier abgetrockneten Präparates in Alkohol absolutus wurden die

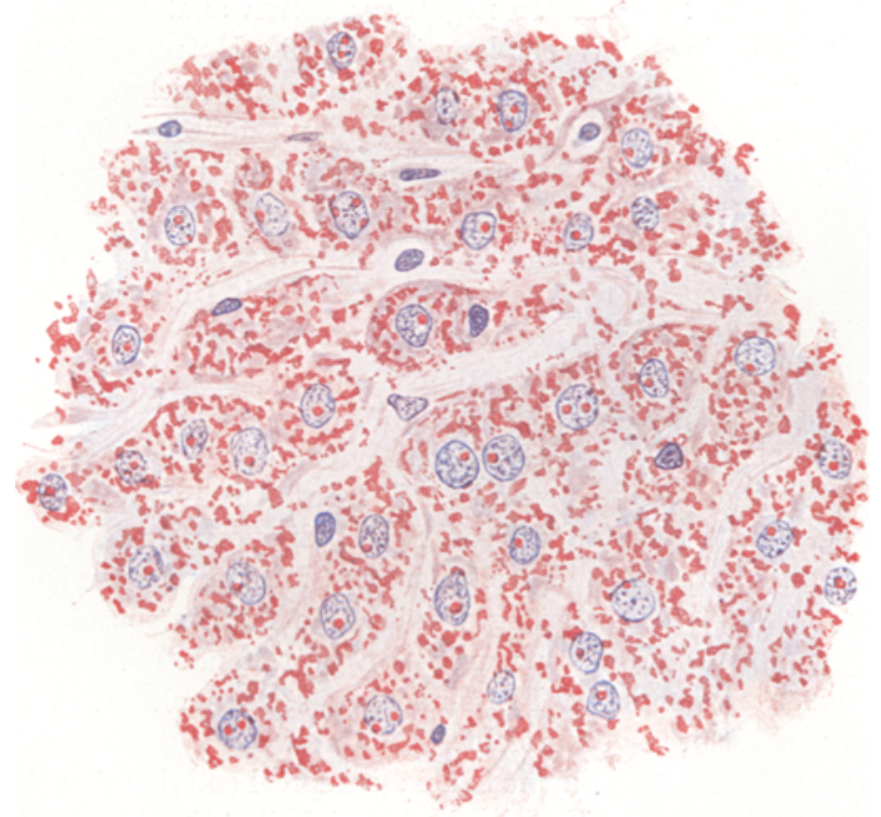

Abb. 1. Leber einer reichlich mit Fleisch gefütterten Ratte. Fix. Alcohol. absol., Paraffinschn. $5 \mu$ Methylgrün-Pyronin. $Z$ e is s, apochrom. Immers. 2 mm, num. Ap. 1,3, Zeichenapparat nach A b b e.

Schnitte in Xylol gebracht uıd dann in Canadabalsam eingeschlossen. Da die Methylgrün-Pyroninfärbung gegen Säure sehr empfindlich ist, wurde ansschließlich ein in Xylol gelöster Canadabalsam verwendet, der speziell zur Benutzung nach dieser Färbung von der Firma Dr. Grü bler \& Co. in Leipzig geliefert wird.

Betrachtet man auf diese Weise angefertigte Präparate der Leber von Ratten, die nach einwöchiger Fleischfütterung geschlachtet wurden, so erhält man folgendes Bild (Abb. 1): Die Leberzellen sind an sich groß, ihre Grenzen verhältnismäßig undeutlich. Die Lebercapillaren sind eng. Auch die Zellkerne sind groß; neben dem grün gefärbten Chromatinnetz 
tritt stets das mit Pyronin leuchtend rot gefärbte Kernkörperchen deutlich hervor. Von der gar nicht oder nur ganz blaßrosa gefärbten Substanz des Zellkörpers heben sich die von Berg beschriebenen Produkte der Eiweißspeicherung als schollen- oder tropfenförmige Gebilde von unregelmäßiger Form und Größe ab; diese Eiweißschollen sind ebenso leuchtend rot gefärbt wie die Kernkörperchen. Meist liegen sie isoliert voneinander, doch gewahrt man häufig auch eine Verbindung einzelner Körper durch rot gefärbte Substanzbrücken. In vier Fütterungsversuchen zeigten sich gänzlich übereinstimmende Bilder. Vergleicht man hiermit Leberpräparate von Tieren, die nicht mit besonders eiweißreichem Futter ernährt wurden (Semmelfütterung), so gewahrt man unter Umständen überhaupt keinen Unterschied. Bei näherer Betrachtung - es wurden im ganzen acht Kontrolltiere untersucht - finden sich jedoch geringe, wenn auch sehr charakteristische Unterschiede zwischen vorwiegend mit Fleisch und vorwiegend mit Semmel gefütterten Tieren und Unterschiede zwischen den einzelnen Tieren der letzteren Kategorie, wobei man jedoch immer verschiedene Gesichtsfelder eines Präparates, besser noch außerdem verschiedene Schnitte desselben Leberstückes zum Vergleich heranziehen muß. Die mit Pyronin rot gefärbten Körper sind bei deh vorwiegend mit Semmel gefütterten Tieren der Zahl nach spärlicher. Sie füllen dann nicht die ganze Zelle aus, sondern finden sich hauptsächlich in der Umgegend des Kernes. Auch ihre Größe ist geringer als nach reichlicher Fleischfütterung. Dabei ändert sich oft zugleich die Form, indem die Körper aus der mehr oder weniger kugeligen Schollen- und Tropfenform zuweilen in eine längliche Stäbchenform übergehen. In einem solchen Präparat können sich einmal spärlich große oder spärlich kleine rot gefärbte Körper oder aber auch reichlich kleine rot gefärbte Körper vorfinden. Diese verschiedenen Möglichkeiten sind in den verschiedenen Zellen ein und derselben Leber zuweilen alle nebeneinander vertreten, so daß man oft an einem Schnitt sehr wechselnde Bilder sehen kann; andererseits verhalten sich die Zellen eines und desselben Leberläppchens in der Regel alle gleichmäßig.

Während man also bei reichlich mit Eiweiß gefütterten Tieren in allen Leberzellen gleichmäßig dasselbe Bild hat: zahlreiche, große, schollenförmige Körper, kann ein vorwiegend mit Semmel gefüttertes Tier verschiedene Bilder zeigen. Unter diesen ist jedoch das zuerst beschriebene: große schollenförmige Körper, aber in geringerer Zahl als beim mit Eiweiß gefütterten Tier, das bei weitem am häufigsten, typische.

Untersucht man die Leber von Hungerratten, so beobachtet man ganz charakteristische Veränderungen an den „Eiweißschollen“" in dem Sinne, daß die Eiweißschollen immer spärlicher und weiterhin immer kleiner werden. An Stelle kompakter mehr oder weniger kúgeliger Schollen treten oft die stäbchenförmigen Körper auf, die sich hier und da auch schon 
beim mit Semmel gefütterten Tiere zeigen. Zuweilen erscheint beim Hungertier die stark mit Pyronin färbbare Substanz nur noch in Form einer feinen, staubartigen Verteilung im Zellkörper (vgl. hierzu Abb.2). Im äußersten Falle ist schließlich alle stark mit Pyronin färbbare Substanz verschwunden.

In dem Maße als beim Hurgertier die „Eiweißschollen“ an Zahl und Größe abnehmen, ändert aber auch die übrige Substanz des Zelleibes ihr Verhalten in bezug auf die Färbbarkeit mit Pyronin. Die Grundsubstanz des Zelleibes, die beim normalen Tier mit Pyronin gar nicht oder nur ganz schwach rosa gefärbt wird, nimmt allmählich eine immer intensivere Rosafarbe an, erreicht aber auch in den äußersten Fällon niemals den leuchtend roten Farbton der „Eiweißschollen", so daß Verwechslungen ausgeschlossen sind. Bei einem verhungerten Tier erscheint schließlich der Zellkörper als aus einer gleichmäßig kräftig rosa gefärbten, mehr oder weniger stark vakuolisierten Substanz bestehend.

Neben diesem allmählichen Verschwinden der „Eiweißschollen“ werden jedoch auch andere histologische Veränderungen an der Leber im Hungerzustande bemerkbar. Die. Zellen werden stark verkleinert, und zwar beteiligt sich hieran sowohl der Zelleib als in geringerem Maße auch der Zellkern. In fortgeschrittenem Hungerzustande bekommt letzterer eine mehr oder weniger gerunzelte Oberfläche. Das Chromatingerüst wird stärker färbbar und zeigt mehr dicke Körnchen anstatt feinerer Fäden. Dabei finden sich verhältnismäßig mehr Kerne mit 2, ja auch 3 Kernkörperchen als beim normalen Tier, wo vorwiegend Zellen mit nur 1 Kernkörperchen vorhanden sind. Je länger das Tier gehungert hat, um so weiter wird das Lumen der Capillaren auf Kosten der Zellgröße (Abb. 3). Dementsprechend sieht man makroskopisch an der frischen Leber, daß die Leber des Hungertieres bedeutend blutreicher ist als die des normalen und an Stelle der normalen rotbraunen eine dunkelblaurote Färbung annimmt.

In einem Falle war bereits nach einer Hungerzeit von 8 Stunden eine deutliche Abnahme der „Eiweißschollen“" sowohl hinsichtlich ihrer Zahl als ihrer Größe nach zu bemerken. In einem anderen Versuche verhungerte eine ausgewachsene Ratte binnen $3 \frac{1}{2}$ Tagen (84 Stunden); eine Hungerzeit von 8 Stunden ist demnach für die Ratte schon verhältnismäßig lang, dürfte aber noch nicht Veränderungen in der Leber bedingen, die als abnorm zu bezeichnen sind, da die zahmen weißen Ratten, die im Laboratorium in Käfigen gehalten wurden, hauptsächlich bei Nacht fraßen und tagsüber oft 8 Stunden und länger kein Futter zu sich nahmen, obwohl ihnen solches zur Verfügung stand.

In voller Übereinstimmung mit Berg ergeben also die bis jetzt mitgeteilten Versuche an Ratten, daß in den Leberzellen schollen- oder tropfenförmige, stark mit Pyronin färbbare Gebilde vorkómmen, die 
nach Eiweißfütterung besonders groß und reichlich auftreten, im Hungerzustande allmählich immer spärlicher und kleiner werden, bis sie sehließlich verschwinden.

Desgleichen konnte ich die Angabe von Berg bestätigen, daß die Schollen sich mit Millonschem Reagens rosa färben. Zu diesem Zwecke wurden auf Objektträger aufgezogene entparaffinierte Schnitte einige Minuten in Millonschem Reagens erwärmt. - Von Pepsinsalzsäure werden die Schollen vollkommen verdaut. Diese Befunde sind nicht anders zu deuten, als daß die Schollen ganz oder wenigstens im wesentlichen aus Eiweißkörpern bestehen müssen.

Die Eiweißsschollen werden auch von anderen Plasmafarbstoffen als von Pyronin gefärbt. So erhält man z. B. auch zum Vergleich der bei Eiweißfütterung und im Hungerzustand auftretenden Veränderungen brauchbare Präparate, wenn man mit Eosin und Hämalaun nach P. Ma yer färbt. Jedoch ist das Pyronin für unseren Zweck vorzuziehen, da diesem Farbstoff ein elektives Färbevermögen für die Schollen zukommt.

Im Hinblick auf weiterhin mitzuteilende Versuche wurde außerdem die an und für sich unwahrscheinliche Möglichkeit geprüft, ob die Schollen vielleicht im wesentlichen nicht aus Eiweiß, sondern aus Glykogen bestehen könnten. Zu diesem. Zwecke wurde ein Paraffinschnitt eines Leberstückes genommen, das einer reichlich mit Eiweiß gefütterten Ratte entstammte, und mit wässeriger Jodjodkaliumlösung behandelt, worauf in allen Zellen die charakteristische Reaktion auf Glykogen eintrat, indem große, braunrote, fast den ganzen Zellkörper erfüllende Schollen sichtbar wurden. Hierbei stellte sich heraus, daß die Jodreaktion erheblich deutlicher ausfiel und auch in Zellen die Anwesenheit von Glykogen anzeigte, in denen sonst kein Glykogen nachweisbar war, wenn man die durch Xylol entparaffinierten und durch absteigenden Alkohol in Wasser geführten Schnitte nicht sofort, sondern erst nach etwa 10 Minuten langem Erwärmen in destilliertem Wasser von 80-90 ${ }^{\circ}$ mit Jodjodkaliumlösung behandelte. Das Glykogen wird somit durch die Erwärmung noch nicht oder wenigstens noch nicht in wesentlichen Mengen aus dem Schnitt herausgelöst, sondern vielmehr in einen reaktionsfähigeren Zustand (Quellung?) übergeführt.

Weitere Schnitte desselben deutlich Glykogenreaktion gebenden Leberstückes, von denen ein Teil vorher in der üblichen Weise mit Methylgrün-Pyronin gefärbt worden war, wurden nun eine halbe Stunde lang bei $37^{\circ}$ mit frischem, filtrierten, unverdünnten Speichel behandelt. Hierauf zeigte sich, daß die vorgefärbten Schnitte ihre Färbung nach der Speichelverdauung nicht verändert hatten. Von den nicht vorgefärbten Schnitten wurde nach halbstündiger Speichelverdauung ein Teil der Jodreaktion unterworfen, der andere mit Methylgrün-Pyronin gefärbt. 
Während bereits nach halbstündiger Speichelverdauung die Jodreaktion gänzlich negativ ausfiel, blieb die Färbbarkeit der Schollen mit Methylgrün-Pyronin bestehen. Allerdings darf man die Verdauung nicht viel länger bei Körpertemperatur ausdehnen, da dann die Färbbarkeit mit Methylgrün-Pyronin verloren geht. Nicht nur die Schollen des Zelleibes, sondern auch die Kernkörperchen lassen sich dann nicht mehr mitPyronin färben, bzw. sie verlieren ihre leuchtende Farbe, wenn es sich um vorgefärbte Präparate handelt. Jedoch bleiben die Schollen auch nach 24stündiger Behandlung mit Speichel bei $37^{\circ}$ noch als stärker lichtbrechende Differenzierungen des Zellkörpers deutlich wahrnehmbar.

Aus diesen Versuchen geht hervor, daß die mit Pyronin färbbaren Schollen nicht aus Glykogen bestehen. Das Glykogen erscheint in anderer Verteilung, nämlich in Form bedeutend größerer Schollen als die mit Pyronin fürbbare Substanz in der Zelle. Das Glykogen wird durch Speichel rasch verdaut, während die mit Pyronin färbbaren Schollen nicht verdaut werden. Ferner ließ sich feststellen, daß Präparate, deren Zellen alle reichlich mit Pyronin färbbare Schollen enthielten, unter Umständen fast frei von Glykogen waren, soweit sich dies durch die Behandlung mit Jodjodkaliumlösung vor und nach Erwärmung des Schnittes nachweisen läßt.

In den meisten Fällen wurde sofort nach Tötung des Versuchstieres ein frisches Zupfpräparat der Leber in Ringerlösung bei starker Vergrößerung untersucht. Deutliche Unterschiede im Aussehen der Leberzellen zwischen Präparaten von nach vorwiegender Fleischfütterung und Präparaten von nach vorwiegender Semmelfütterung getöteten Tieren ließen sich nicht feststellen. In beiden Fällen sind die Zellen prall mit den bekannten mehr oder weniger stark lichtbrechenden und mehr oder weniger großen Granula angefüllt. Die Zellgrenzen sind wenig deutlich, ebenso tritt der Kern njeht sehr deutlich hervor. Im vorgeschrittenen Hungerzustande sind die Zellen verkleinert, Zellgrenzen und Kern treten schärfer hervor, aber auch hier zeigen die Granula des Zellkörpers keine besonders deutlichen Unterschiede in bezug auf Größe und Verteilung gegenüber der normalen Zelle. Ebensowenig wurde durch Eiweißfütterung oder Hunger eine charakteristische Veränderung des Zelleibes bei Fixierung und Säurefuchsinfärbung der Leber nach dem Altmannschen Granulaverfahren bemerkbar.

Man könnte auf Grund der Untersuchungen von Berg die Meinung gewinnen, daß das von ihm meines Erachtens einwandfrei in den Leberzellen nachgewiesene Reserveeiweiß auch in der lebenden Zelle in Form von ,Tropfen" enthalten ist, ebenso wie es uns am fixierten und mit Pyronin gefärbten Präparat erscheint. Auf Grund der Befunde an frischen Zupfpräparaten und Altmann -Präparaten kann ich dieser Anschauung nicht beipflichten. Bei Betrachtung dieser Präparate 
drängt sich sofort unwillkürlich die Frage auf, wo die ,Eiweißschollen“ in der mit Granula dicht erfüllten Zelle noch Platz haben. Man wird um so mehr geneigt sein, die ,Eiweißschollen“ in gewissem Sinne als Kunstprodukte anzusehen, als sich zwischen den frischen Leberzellen von vorwiegend mit Eiweiß und vorwiegend mit Semmel gefütterten Tieren keinerlei charakteristische Unterschiede wahrnehmen lassen.

Es ist viel wahrscheinlicher, daß die ,Eiweißschollen" Produkte einer durch die Fixierung hervorgerufenen Gerinnung besonderer Eiweißkörper sind, und es läßt sich nicht entscheiden, ob die Eiweißschollen aus Bestandteilen der Granula der lebenden Zellen oder aus der mehr flüssigen intergranulären Substanz des Protoplasmas hervorgegangen sind. Damit wird der Wert und die Bedeutung der Pyroninfärbung als einer mikrochemischen Reaktion auf Eiweißspeicherung in den Leberzellen keineswegs herabgesetzt.

Auf jeden Fall läßt sich mit Hilfe der Pyroninfärbung nachweisen, daß tatsächlich Eiweißkörper in der Leber gespeichert werden, ebenso wie dies für die Speicherung von Kohlenhydraten in Form von Glykogen schon lange bekannt ist. Es liegt nun nahe, sich die Frage vorzulegen, unter welchen Umständen dieses „Reserveeiweiß“ der Leber wieder mobilisiert und dem Kreislauf zugeführt wird. Daß diese Mobilisierung im Hungerzustande ebenso wie beim Glykogen eintritt, ist bereits durch die Versuche von Berg erwiesen.

Wenn man feststellt, daß das Glykogen der Leber im Hungerzustande von den Zellen wiederum zu Traubenzucker zerlegt und dem Kreislauf zugeführt wird, so ist damit noch nichts über die eigentliche Ursache oder besser über den Reiz, auf Grund dessen diese Mobilisierung des Glykogens erfolgt, ausgesagt. Bis jetzt wissen wir nur, daß das Glykogen der Leber durch Adrenalin mobilisiert werden kann. Es erhebt sich nun die Frage, ob der Reiz, den das Adrenalin auf die Leberzellen ausübt, nicht nur eine Mobilisierung des Glykogens, sondern auch eine Mobilisierung des Reserveeiweißes der Leberzellen zur Folge hat.

Um diese Frage zu prüfen, wurde in einer Reihe von Versuchen Ratten subcutan Adrenalin eingespritzt, und zwar sowohl Ratten, die vorwiegend mit Fleisch (5 Versuche) als solchen, die vorwiegend mit Semmel gefüttert waren (6 Versuche). Dann wurden die Ratten, sofern die verabreichte Adrenalinmenge nicht tödlich war, nach einer Reihe von Stunden (3-8) geschlachtet und die Leber in der beschriebenen Weise fixiert, geschnitten und gefärbt. Zur Verwendung gelangte Suprareninum hydrochloricum synthetic. Höchst im Verhältnis von $1: 1000$ in 0,9 proz. Kochsalzlösung gelöst. Die verabreichte Menge, die bei subcutaner Anwendung des Adrenalins bedeutend höher sein kann als bei intravenöser ${ }^{1}$ ), wurde auf das Gramm Körpergewicht berechnet. Bei dieser Anwendung

1) Vg1. hierzu Biedl, Innere Sekretion, 1, 521. 2. Aufl., 1913. 
liegt die Maximaldosis, die eben noch vertragen wird, bei etwa $0,005 \mathrm{mg}$ pro Gramm Körpergewicht. In einem scheinbar atypischen Falle starb das Versuchstier nach Injektion von nur 0,004 mg pro Gramm Körpergewicht bereits nach einer halben Stunde unter allgemeinen Krämpfen. Wurden höhere Dosen als 0,005 gm pro Gramm Körpergewicht verabreicht, so starben die Tiere nach 4-5 Stunden. Oft schon wenige Minuten nach der Adrenalininjektion änderten die Tiere ihr Verhalten, saßen erst ruhig mit gesträubten Haaren im Käfig und zeigten eine sehr angestrengte Atmung. Später trat motorische Unruhe ein, dabei war

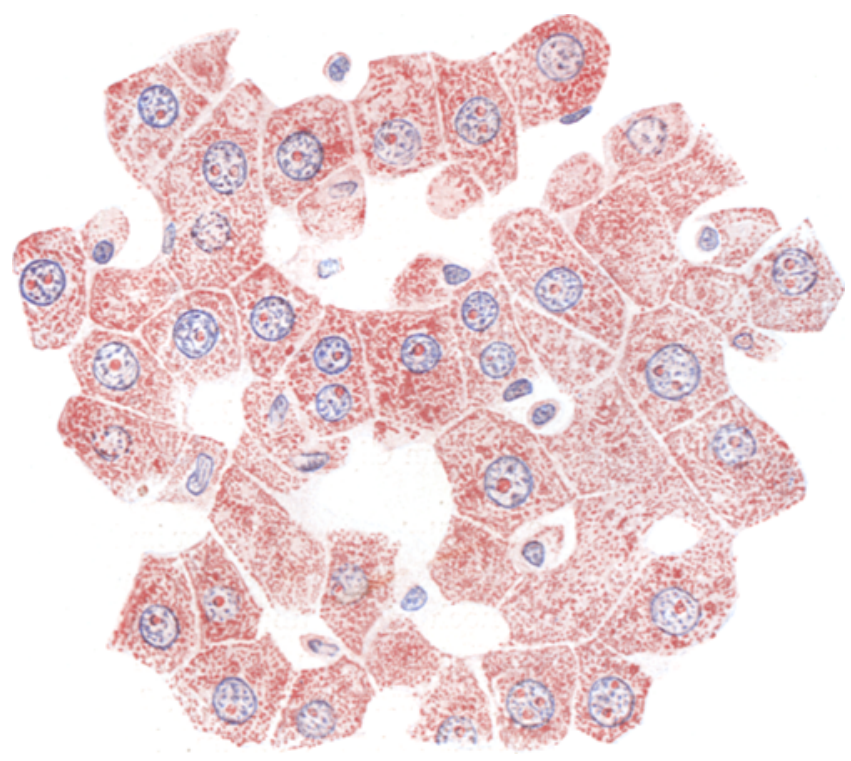

Abb. 2. Leber einer reichlich mit Fleisch gefütterten Ratte 4 Stunden nach Einspritzung von Adrenalin. Technik s. Abb. 1.

aber eine Lähmung der hinteren Körperhälfte zu beobachten. Vor dem Tode wurden die Tiere schließlich apathisch. War die verabreichte Adrenalinmenge nicht tödlich, so waren die soeben beschriebenen Krankheitserscheinungen bedeutend geringer und gingen nach 2-4 Stunden völlig zurück. Wurde den Versuchstieren eine nicht tödliche Menge verabreicht, so wurden sie 3-8 Stunden nach der Einspritzung geschlachtet.

Bei der Sektion der mit Adrenalin behandelten Ratten bestand die auffallendste stets vorhandene Änderung' in einer starken Hyperämie der Leber. Die Leber war dunkelblaurot gefärbt und ihre Gefäße waren prall mit Blut gefüllt.

Das mikroskopische Bild der Leber von mit starken Adrenalindosen (über 0,003 mg pro g Körpergewicht) behandelten Ratten zeigte sehr auf- 
fallende und in sämtlichen. Versuchen übereinstimmende Veränderungen, wenn man die betreffende Leber mit derjenigen eines normalen - sei es vorwiegend mit Semmel oder vorwiegend mit Fleisch gefütterten - Tieres vergleicht. Und zwar verändert sich bereits innerhalb ganz kurzer Zeit (z. B. nach 3 Stunden) die Leber genau in demselben Sinne, wie sie sich im Hungerzustande verändert. Die mit Pyronin leuchtend rot gefärbten Eiweißschollen werden kleiner und spärlicher; gegenüber den

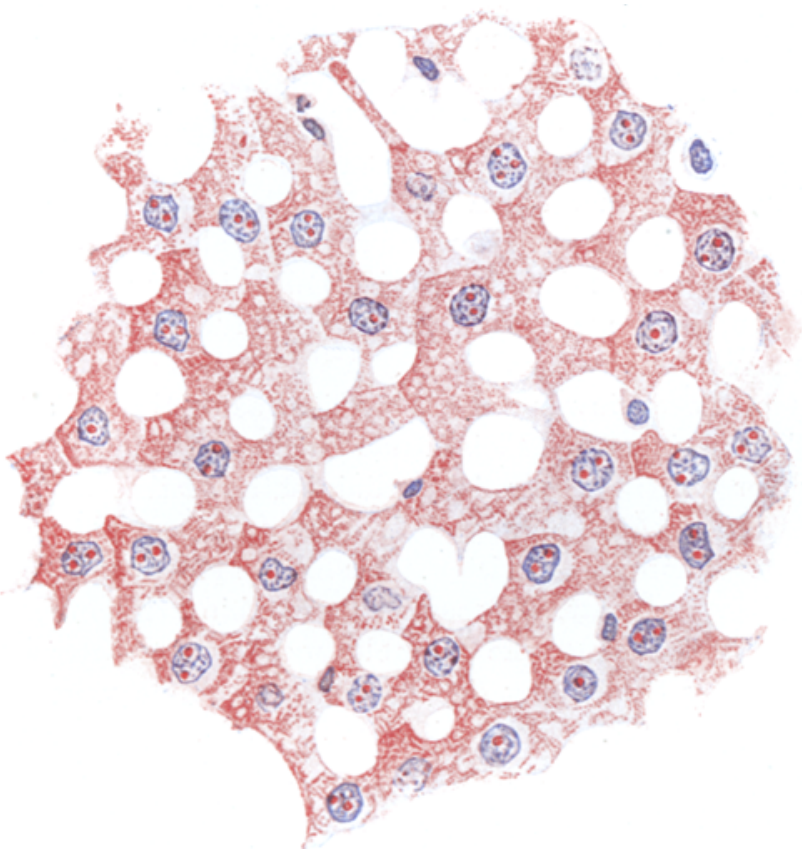

Abb. 3. Leber einer verhungerten Ratte. Technik s. Abb. 1.

kompakten Schollen treten mehr stäbchenförmige Gebilde auf. Weiterhin kommt es zu einer nur noch staubartigen Verteilung (Abb. 2) und schließlich zu einem völligen Verschwinden der mit Pyronin leuchtend rot gefärbten Substanz. In einem Versuche waren nach Einspritzung von nur $0,003 \mathrm{mg}$ Adrenalin pro Gramm Körpergewioht die Eiweißschollen gänzlich verschwunden, so daß in dieser Beziehung die Leber der eines verhungerten Tieres gleichkam (vgl. Abb. 3). In anderen Fällen zeigten sich alle die bei den verschiedenen Stadien des Hungerzustandes vorkommenden Abstufungen in bezug auf Menge und Form der Eiweißschollen. Wurden die mit Adrenalin behandelten Tiere 8 Stunden nach der Einspritzung geschlachtet, so bestand stets ein ganz unverkennbarer Unterschied zwischen der Leber dieser Tiere und derjenigen von 
Tieren, die nach einer einfachen Hungerzeit von 8 Stunden geschlachtet worden waren. Auch die Einspritzung von $1 \mathrm{ecm} 0,9$ proz. Kochsalzlösung ohne Adrenalinzusatz hatte keinen Einfluß auf das mikroskopische Bild der Leberzellen.

Neben der Veränderung der Eiweißschollen fanden sich an der Leber nach Adrenalineinspritzung auch weiterhin die für den Hungerzustand charakteristischen Veränderungen: eine stärkere diffuse Färbbarkeit des Zelleibes, ein Hervortreten der Zellgrenzen, eine deutliche Verkleinerung der Zellen und eine der makroskopisch wahrnehmbaren Hyperämie entsprechende starke Erweiterung der Capillaren. Diese letztere Erscheinung ist bereits an mit Adrenalin längere Zeit behandelten Kaninchen von Grober ${ }^{1}$ ) wahrgenommen worden.

Die Veränderungen an den Eiweißschollen waren an Tieren, die vorwiegend mit Fleisch ernährt worden waren, also vor der Adrenalineinspritzung besonders reichlich Eiweißschollen in ihren Leberzellen besitzen mußten, ebenso stark ausgeprägt als bei Tieren, die vorwiegend mit Semmel gefüttert worden waren.

Um ein möglichst einwandfreies Vergleichsmaterial zu erhalten, wurde bei zwei Tieren, einem mit Fleisch und einem mit Semmel gefütterten, die Leber vor und nach der Adrenalininjektion mikroskopisch untersucht. Es wurde in Äthernarkose die Laparotomie gemacht und ein kleines Stück Leber herausgeschnitten. Der hierbei eintretende Blutverlust ist ganz gering, auch tritt, wie man sich späterhin bei der Sektion überzeugen kann, keine merkliche Nachblutung aus der Leberwunde ein. Nach sorgfältiger Schließung der Laparotomiewunde wurde subcutan Adrenalin eingespritzt. Nach weiteren 3 Stunden wurde das Tier geschlachtet und ein zweites Stück Leber zur mikrnskopischen Untersuchung entnommen. Beide Leberstücken wurden in genau derselben Weise fixiert, eingebettet, geschnitten und gefärbt. Das Ergebnis dieser Versuche war ein völlig eindeutiges. Die normale Leber des mit Fleisch gefütterten Tieres enthielt sehr reichlich, die des mit Semmel gefütterten Tieres in etwas geringerer Menge große Eiweißschollen. Nach der Behandlung mit Adrenalin fehlten bei dem mit Fleisch gefütterten Tier die Eiweißschollen in manchen Zellen vollkommen, in anderen waren sie noch vorhanden, aber nur ganz fein verteilt und in sehr geringer Menge (vgl. Abb. 2). Bei dem mit Semmel gefütterten Tier waren die Eiweißschollen aus allen Zellen gänzlich verschwunden (vgl. Abb. 3). In beiden Fällen waren die Lebercapillaren stark erweitert. Um eine Täuschung auszuschließen, die etwa durch eine Schädigung der Leber infolge der Äthernarkose und der Operation hätte bedingt werden können, wurde außerdem bei zwei weiteren Ratten genau dieselbe Operation ausgeführt,

1) Grober, Zentralbl. f. inn. Med. 1908. 
Die Wirkung des Adrenalins anf das in der Leber gespeicherte Eiweiß. 85 ohne daß darauf Adrenalin eingespritzt wurde. Es zeigte sich hierbei, daß das erste, durch die Operation gewonnene Leberstück und das zweite, nach der 3 Stunden nach der Operation erfolgten Tötung des Tieres gewonnene Leberstück makroskopisch und mikroskopisch keinerlei erkennbare Unterschiede aufwiesen. Eine Beeinflussung des histologischen Bildes der Leber durch Äthernarkose oder Operation war also nicht zu beobachten.

\section{Zusammenfassung.}

Bei der Ratte verschwindet nach subcutaner Einspritzung von Adrenalin das in der Leber gespeicherte mikroskopisch nachweisbare Eiweiß. 